\title{
PROPOSTA DE UM MODELO DE ANÁLISE DE DECISÃO NA MANUTENÇÃO UTILIZANDO O DIAGRAMA DE ISHIKAWA COM O MÉTODO MULTICRITÉRIO PROMETHEE EM EQUIPAMENTOS
}

\author{
Vânio Berlin de Souza \\ Faculdade de Ciências Aplicadas FCA - Universidade de Campinas \\ Rua Pedro Zacaria, 1300 - Jardim Santa Luiza - Limeira/SP - CEP 13.484-350 \\ Vanio.berlin@gmail.com \\ Anibal Tavares de Azevedo \\ Faculdade de Ciências Aplicadas FCA - Universidade de Campinas \\ Rua Pedro Zacaria, 1300 - Jardim Santa Luiza - Limeira/SP - CEP 13.484-3 \\ anibal.azevedo@fca.unicamp.br
}

\begin{abstract}
RESUMO
Neste trabalho se reúne conceitos básicos de confiabilidade, da aplicação do diagrama de Ishikawa na manutenção de equipamentos relacionado com um estudo utilizando a metodologia multicritério conhecida como método PROMETHEE para a tomada de decisão das operações de manutenção levando em conta as melhores ações de tratamento da falha estudada focadas na confiabilidade do equipamento. A proposta deste trabalho é o desenvolvimento de um modelo multicritério para o auxílio das decisões de tratamento das causas raízes diagnosticados pelo diagrama de espinha de peixe (Ishikawa) correlacionando às ações e os critérios nas decisões estratégicas. Para a sua elaboração foram utilizados dados coletados de um trator esteiras para ilustrar a aplicação da metodologia.
\end{abstract}

Palavra-chave: Análise da Decisão; Diagrama de Ishikawa; Estudo de Falhas em Equipamentos; Método PROMETHEE; Gestão da Manutenção.

\begin{abstract}
This work brings together basic concepts of reliability, from the application of Ishikawa diagram in equipment maintenance related to a study using the multicriteria methodology known as PROMETHEE method for decision making of maintenance operations taking into account the best fault treatment actions. focused on equipment reliability. The purpose of this work is the development of a multicriteria model to aid the root cause treatment decisions diagnosed by the fishbone diagram (Ishikawa) correlating the actions and the criteria in the strategic decisions. For its elaboration, data collected from a crawler tractor were used to illustrate the application of the methodology.
\end{abstract}

Keywords: Decision analysis; Ishikawa diagram; Equipment Failure Study; PROMETHEE method; Maintenance management. 


\section{Como Citar:}

SOUZA, Vânio Berlin de; AZEVEDO, Anibal Tavares de. Proposta de um modelo de análise de decisão na manutenção utilizando o diagrama de Ishikawa com o método multicritério PROMETHEE em equipamentos. In: SIMPÓSIO DE PESQUISA OPERACIONAL E LOGÍSTICA DA MARINHA, 19 de Novembro de 2019, Rio de Janeiro, RJ. Anais [...]. Rio de Janeiro: Centro de Análises de Sistemas Navais, 2019.

\section{INTRODUÇÃO}

Com mudanças freqüentes no cenário mundial impulsionadas pelas alterações no ciclo de vida dos produtos associadas ao aumento das exigências dos clientes, qualidade assegurada e disponibilidade imediata, as organizações lutam para se manter no mercado competitivo buscam: novas alternativas para reduzir seus custos, qualidade, rapidez na entrega, flexibilidade da manufatura e confiabilidade de seus equipamentos. Para Slack, et al. (2009), embora nenhuma operação produtiva seja indiferente a falhas, em algumas é crucial que os produtos e serviços não falhem - aviões em vôo, fornecimento de eletricidade a hospitais, funcionamento dos cintos de segurança nos carros, equipamentos em reatores nucleares, e outros serviços críticos de emergência por exemplo. Nessas situações, a confiabilidade não é somente desejável, mas essencial, em outras situações, ter produtos e serviços confiáveis pode ser uma forma das organizações conquistarem o sucesso.

E uma forma de aumentar a eficiência do sistema de confiabilidade em equipamentos é através dos estudos de falhas com a utilização do diagrama de Ishikawa para análise das causas raízes que é muito utilizando no auxilio da qualidade e da confiabilidade sendo uma ferramenta essencial na caixa de ferramentas de confiabilidade. Ela evita nas instalações futuras instâncias de falha rastreando a causa raiz dos eventos como impactos de segurança, saúde, meio ambiente, confiabilidade ou produção, em vez de somente corrigir a causa ou a falha imediata. A análise de causa raiz compartilha muitos recursos com o FMEA (Modos de Falha e Análise de Efeitos), com uma grande diferença: um FMEA é realizado antes de eventos de falha usando equipe com experiência, histórico de instalações e ativos, manuais do fabricante, contexto operacional para determinar as causas dominantes de falhas e desenvolver estratégias antecedentes. É uma estratégia proativa destinada a reduzir a incidência de falhas antes que aconteçam. Por sua vez segundo Duphily (2014), a análise de causa raiz é uma estratégia reativa que trata das causas de falha depois que elas acontecem. Nem todas as falhas serão detectadas por um FMEA, pois, novas tecnologias, novos ativos ou alterações de processo podem limitar informações disponíveis sobre seus modos dominantes. Determinar a causa raiz de uma falha requer uma equipe experiente, evidências objetivas, levantamento de informações cruciais, investigação de uma variedade de causas potenciais, dos fatos, o comprometimento da administração, da equipe e percepção nascida da experiência com análises das causas raízes (LORENZO, 2008).

Quanto à Pesquisa Operacional, ciência que auxilia e contribui na análise da decisão pode ajudar os tomadores de decisão na escolha de alternativas com vários critérios (GOMES; GOMES, 2012). Dentre algumas correntes teóricas de análise da decisão destacam-se a Teoria de Apoio Multicritério à Decisão (AMD) e a teoria de jogos (GOMES; GOMES, 2012). Assim este artigo apresenta uma proposta de modelo com aplicação do diagrama de Ishikawa correlacionado com ferramentas de pesquisa operacional para solucionar problemas multicriterios na gestão da manutenção industrial (decisões baseado no risco de um evento com causas múltiplas associadas com diferentes pesos para cada uma).

\subsection{OBJETIVO GERAL}

O objetivo geral do estudo é o desenvolvimento de um modelo que propõe a melhor 
tomada de decisão na gestão da manutenção de equipamentos industriais analisando as causas raízes com a aplicação do diagrama de Ishikawa, propondo a possíveis ações baseado nos impactos operacionais e na confiabilidade utilizado o método multicritério PROMETHEE.

\subsection{OBJETIVO ESPECífFICo}

Como objetivos específicos, aplicam-se neste trabalho o uso de ferramentas da pesquisa operacional correlacionada com ferramentas da qualidade e manutenção para fomentar a interação de multi-áreas de conhecimento em estudos/ações em problemas de engenharia de produção na análise de decisão operacional. Para a execução pretende-se:

Utilizar dados de manutenção de equipamentos (através da parceria de empresas), como as falhas, freqüências, causas, entre outros dados;

Aplicar o diagrama de Ishikawa para diagnosticar as causas raízes encontradas;

Desenvolver um modelo que se correlaciona com a aplicação do diagrama de Ishikawa na manutenção de máquinas/equipamentos com o uso do Método Multicritério PROMETHEE nas decisões estratégicas de manutenção.

Realizar o ranqueamento das possíveis ações de manutenção para mitigação das causas de falha através de método multicritério PROMETHEE.

\subsection{JUSTIFICATIVA}

As indústrias do século XXI enfrentam desafios para otimizar seus sistemas de produção devido ao aprimoramento de tecnologias globais, da competitividade, requisitos ambientais, de segurança e a percepção de qualidade total com diferentes aspectos que desafiam os lucros das empresas. O impacto da manutenção no aspecto do desempenho do negócio, como a produtividade e rentabilidade, aumentaram indefinidamente devido ao seu papel na garantia e melhoria da disponibilidade, eficiência de máquina, qualidade do produto, requisitos de entrega, ambientais e de segurança. Pois a integridade dos equipamentos passou a ser "questão estratégica", visto que a indisponibilidade operacional pode representar em custos, mais do que custaria reparar a própria falha (KARDEC; NASCIF, 2013).

Este estudo é fruto das exigências de padrões aceitáveis de disponibilidade (através da eliminação de pontos de falha do projeto, redundância, dispositivos para prevenir e realizar a manutenção dos seus equipamentos etc.), qualidade, segurança na manutenção e devido à sofisticação dos equipamentos ou dispositivos mecânicos, elétricos, eletrônicos entre outros utilizados nas máquinas atuais, como os veículos e equipamentos inteligentes (smart products) tendo agora maior grau de exigência em confiabilidade.

\section{GERENCIAMENTO DA MANUTENÇÃO}

Sabe-se que o principal elemento de um sistema de gerenciamento da manutenção está na elaboração de um sistema padrão de manutenção, de detecção associado com um sistema de tratamento de falhas e que o plano de manutenção tem uma posição de destaque bem como as metas de melhoria. A elaboração e o cumprimento deste plano permitirão que a empresa atinja seus objetivos de lucratividade por meio de equipamentos que não apresentem falhas, prejudique a qualidade, o custo, a entrega dos produtos, serviços, que não coloquem em risco a segurança e a integridade do meio ambiente. Enfim, o principal objetivo da manutenção é evitar a ocorrência de falhas e isto está expresso na sua missão (XENOS, 2004).

No caso de equipamentos críticos o acompanhamento sistemático do desempenho e os cálculos dos custos operacionais, durante a vida útil, são fatores fundamentais. E isso é um problema complexo que exigem conhecimentos da área econômica e de engenharia. 
Devido a isso fabricantes de equipamentos estão em constante busca pela qualidade, pois o aumento da confiabilidade dos produtos pode representar reputação imediata, bem como competitividade, fidelidade a marca, baixo custo de garantia, suporte e precisão no planejamento de peças de reposição. Por sua vez, os consumidores buscam a qualidade como forma de atingir alto desempenho, disponibilidade, previsibilidade e segurança na operação. Percebe-se então, a necessidade de se fazer uso e ampliar os conhecimentos de confiabilidade em equipamentos, para que velhas lições se renovem e evitem a repetição de falhas conhecidas. As decisões devem ser analisadas baseadas em critérios direcionadores do planejamento estratégico da instituição e critérios financeiros. Portanto, um programa de manutenção adequado, elaborado e acompanhado pelo departamento da gestão da manutenção deve definir diferentes estratégias de manutenção para máquinas diferentes.

Para construir o referencial teórico e estabelecer uma base solida a fim de consolidar e orientar o objetivo do artigo, os próximos tópicos foram desenvolvidos com foco de esquadrinhar, esclarecer, propor e respaldar a pesquisa proposta.

\subsection{OS MODELOS DE FALHAS}

Basicamente a freqüência de ocorrência de falhas para um equipamento pode variar de três maneiras diferentes ela pode ser: constante, crescente ou decrescente.

Uma freqüência constante é característica de equipamentos cujas falhas são causadas por eventos aleatórios, resultando na aplicação de esforços que excedem a resistência do equipamento como esforços excessivos devido a sobrecargas acidentais, erros de manutenção e operação que ocorrem numa razão constante, ou seja, a probabilidade de tais ocorrências não tende a variar à medida que o equipamento envelhece (XENOS, 2004).

Uma probabilidade de ocorrência crescente é típica de situações de fadiga de materiais, corrosão ou desgaste (a probabilidade de ocorrência de falhas aumenta à medida que o equipamento envelhece). Várias partes dos equipamentos se comportam desta maneira, principalmente aquelas que se deterioram naturalmente com o tempo, com a carga aplicada que estão sujeitas a esforços cíclicos e repetitivos.

Finalmente, uma probabilidade de ocorrência decrescente é característica de equipamentos cuja confiabilidade intrínseca aumenta com o tempo, como no caso da introdução de melhorias nos equipamentos, implicando a substituição dos componentes e peças por outros mais confiáveis. Além disso, a freqüência de ocorrência de falhas tende a diminuir no início da vida útil dos equipamentos, quando os problemas de projeto, fabricação e instalação vão sendo gradualmente eliminados. O efeito combinado destes três modelos de falhas dá origem ao modelo de falhas chamado de curva da banheira, devido ao seu formato característico (KARDEC; NASCIF, 2013).

O próximo tópico descrito será o modo de tratamento sistêmico de forma que seja sanada permanente (se possível). Para isso decisões de manutenções (seleção de uma opção entre várias alternativas baseada na preferência, inferência, classificação e julgamento, quer consciente ou inconsciente) serão necessários a fim de obter o melhor resultado: eficiência das atividades de manutenção, diagnóstico preciso da falha além da disposição de equipamento com o custo apropriado.

\subsection{A TOMADA DE DECISÃO NA MANUTENÇÃO}

A tomada de decisão na manutenção geralmente será o produto de uma interação entre as preferências de stakeholders em um interesse comum na decisão e intervirão para afetá-la diretamente através dos sistemas de valores que possuem descritas em sua política. E a definição de qual política de manutenção se aplica à determinado equipamento, para reduzir os custos e aumentar a eficiência, é de grande importância a partir da necessidade de um processo estruturado em que se identifique as variáveis e critérios mais relevantes, as 
atividades de manutenção aplicadas e seus detalhes devem ser considerados (KARDEC; NASCIF, 2013).Os custos, segurança, recursos, viabilidade, disponibilidade, confiabilidade, riscos e impacto ao meio ambiente são critérios que cada um poderá resultar em uma ou mais possíveis políticas de manutenção sugeridas (manutenção corretiva, preventiva ou preditiva) e torna-se um macro processo estruturado de tomada de decisão. Quanto à política de manutenção; ressalta-se que o processo proposto deve ser alinhado às especificidades da organização, logo, o mesmo serve como referência, levantando questionamentos relevantes à tomada de decisão e direcionando a organização (RODRIGUES; SELEME; CLETO, 2015).

Desta forma, aspectos relevantes de manutenção do ativo foi estudado e sua importância valorizada para otimização do resultado do processo de tomada de decisão.

\subsection{A ANÁLISE DA QUALIDADE DA MANUTENÇÃO COM O DIAGRAMA DE ISHIKAWA AUXILIANDO A CONFIABILIDADE DE EQUIPAMENTOS INDUSTRIAIS}

A análise da cadeia de causa e efeito com o diagrama de Ishikawa provou ser uma das ferramentas mais populares por uma série de razões: seus princípios são fáceis de aprender e usar, é extremamente flexível na medida em que pode ser aplicado a uma variedade de problemas de natureza diferentes, podem penetrar nas raízes profundamente ao tamanho dos átomos se necessário - onde outras ferramentas muitas vezes param, e seus resultados são fáceis de comunicar (DOBRUSSKIN, 2016).

Para os autores Ben-Daya; Duffuaa (1995), o relacionamento qualidade e manutenção acontecem porque o aspecto da qualidade é sentido através da relação definida entre manutenção e produção, ou seja, um equipamento mal mantido quebra ou falha e produz baixa qualidade sendo detectado pelo controle de qualidade e ações de manutenção são necessárias. Portanto, é possível vincular a manutenção e a qualidade na determinação dos limites de tolerância abaixo dos quais a qualidade não é "satisfatória". O objetivo é manter (com qualidade nas ações) o equipamento em ótimo estado para suprir a capacidade de fabricação mantendo a qualidade. E a manutenção produtiva total (TPM) foca na relação da manutenção e qualidade (DEHOMBREUX, 2012).

\subsubsection{Diagrama de Ishikawa}

O diagrama de Ishikawa é um método efetivo para encontrar as causas raízes de um problema (SLACK et. al., 2009). Ele inicia-se considerando o efeito, mostrado à direita na Figura 01; este efeito pode ser um problema relacionado à qualidade, uma situação desejada, ou qualquer condição descrita claramente. Segundo (ISHIKAWA, 1993), as palavras que aparecem nas pontas das ramificações do diagrama da Figura 01 são as famílias de causas, que podem ser classificadas como matérias-primas, máquinas, medidas, meio ambiente, mão-de-obra e método - os chamados $6 \mathrm{M}^{\prime}$ s. Outras famílias de causas podem ser utilizadas nas pontas das ramificações, dependendo do problema investigado. Porém, o uso da categorização utilizando os 6M's é mais comum, mesmo que em alguns casos nem todos sejam utilizados (SLACK et. al., 2009). Para as causas listadas fazem-se perguntas: o que, onde, como e por que, mas acrescentando algumas “respostas” possíveis de forma explicita.

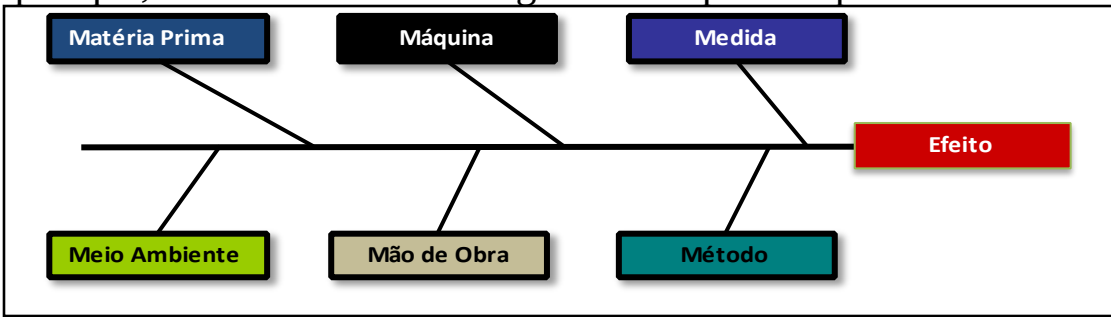

Figura 01 - Diagrama de Ishikawa. Fonte: Adaptado pelo autor de Ishikawa, 2019.

O diagrama pode ser entendido como uma representação de processos/subprocessos; o efeito em ambos é provocado pelas causas, que são classificadas dentro dos $6 \mathrm{M}$ 
's, em seguida ocorre à busca dentro de cada $\mathrm{M}$, como em um brainstorming orientado. $\mathrm{O}$ diagrama é muito utilizado para mostrar várias causas potenciais de defeitos em produtos e suas inter-relações, sendo útil em resumir conhecimento do processo (ISHIKAWA, 1993). Ele organiza as causas relacionadas a um efeito específico que se deseja estudar, uma vez que sempre que um efeito (resultado) ocorre, há um conjunto de causas (meios) que podem ter influência.

\subsection{O MÉTODO MULTICRITÉRIO PROMETHEE}

O método Promethee é um método de superação, que consiste em construir uma relação binária muito particular entre as alternativas em análise (Preference Ranking Organization Method for Enrichment Evaluations) foi dessenvolvido pela escola francesa de tomada de decisão desenvolvida pelos professores J.P. Brans, B. Mareschal e P. Vincke, em 1984, e aperfeiçoado desde então, (BRANS, 1994), (BRANS, 2002). A escolha desse método foi resultado da sua objetividade e flexibilidade. Consideramos que a sua utilização rápida, a fácil verificação, a transparência nos resultados e a flexibilização do processo de comparação foram fatores fundamentais na escolha do método. É uma ferramenta que tanto pode ser aplicada em grupos como individualmente. Atribuindo a cada critério um peso proporcional à sua importância, calcula-se, para cada par (a, b) de ações, o grau de superação.A cada simulação realizada, o Promethee II define uma pontuação cardeal para cada alternativa existente, que pode ser usada para desenvolver uma completa ordenação destas. Comparando as alternativas a e b, o grau de sobre classificação $\pi(a, b)$ é:

$$
\pi(a, b)=\frac{1}{W} * \sum_{j=1}^{n} w_{j} * F_{j}(a, b) \text {, onde } W=\sum_{j=1}^{n} w_{j}
$$

Sendo $w_{j}$ o peso do critério j, variando de 1 até n, com o maior peso sendo atribuído ao critério de maior importância. $F_{j}(a, b)$ é a função do critério, chamada de "função preferência". Estas funções assumem valores entre 0 e 1, associadas a cada critério, indicando a preferência entre alternativas, e são representadas em função da diferença do critério perante as alternativas, sendo escolhidas conforme o problema em conjunto com o decisor. Para estimar-se $F_{j}(a, b)$ é oferecida a ele a escolha, para cada critério, entre seis formas de curvas conhecidas (critério usual, quase-critério, critério de preferência linear, nivelamento, critério de preferência linear com zona de indiferença, e a curva gaussiana).

A escolha do método a ser empregado depende do tipo de problema em análise, do contexto estudado, dos atores envolvidos, da estrutura de preferência e do tipo de resposta que se deseja alcançar, ou seja, qual a problemática de referência (GOMES, 2002). Dessa forma, o problema em questão, como já caracterizado anteriormente, visa uma priorização de alternativas, sofrendo a influência de vários agentes de decisão por se tratar de investimento que vai refletir em aspectos técnicos, ambientais, materiais e humanos. Cada um desses aspectos será considerado como critério na avaliação, e, portanto, requer uma informação intercritério que corresponda à sua importância relativa. Para esses casos, freqüentemente faz-se uso dos métodos da Escola Francesa que utilizam a abordagem outranking (superação, subordinação, prevalência e sobre classificação) ou surclassement, da terminologia original em francês (ROY, 1985). Estudando os critérios e as ações a serem analisados, os parâmetros a serem buscados podem ser escolhidos entre uma ou mais formas de curvas para se levantar os resultados e o foco do que se quer diagnosticar bem como o estudo destes dados. Para isso deve-se realizar um modelo de simulação pelo método multicritério Promethee, que será abordado no próximo tópico.

\subsubsection{Diagrama de Ishikawa}

Com a complexidade e o crescimento de informações necessárias no processo decisório fez com que surgissem novos métodos de decisão para alcançar melhores resultados na tomada de decisão. Neste sentido, métodos multicritérios de tomada de decisão 
que são vistos como ferramentas matemáticas, eficazes para resolução de problemas em que existem critérios conflitantes (BRANS, 2005). Tais métodos fornecem ao usuário uma classificação e ranqueamento das ações candidatas (SAATY, 1991), (SAATY, 2012).

Para (VINCKE, 1992), a vantagem da utilização de métodos multicritérios ocorre pelo fato de que não há, em geral, decisões que sejam simultaneamente ótimas sob todos os pontos de análise, fazendo com que ocorra desta forma, a seleção da melhor opção possível através de um modelo. E as etapas típicas para o seu desenvolvimento de um modelo de simulação são: a formulação do problema; a definição de objetivos e plano geral do projeto; o seu conceito; a coleta de dados; a tradução do modelo; a verificação; a validação; a definição dos experimentos; a execução e análise; a documentação e desenvolvimento de relatórios; por fim a implementação. A Figura 02 ilustra a montagem de um sistema decisório.

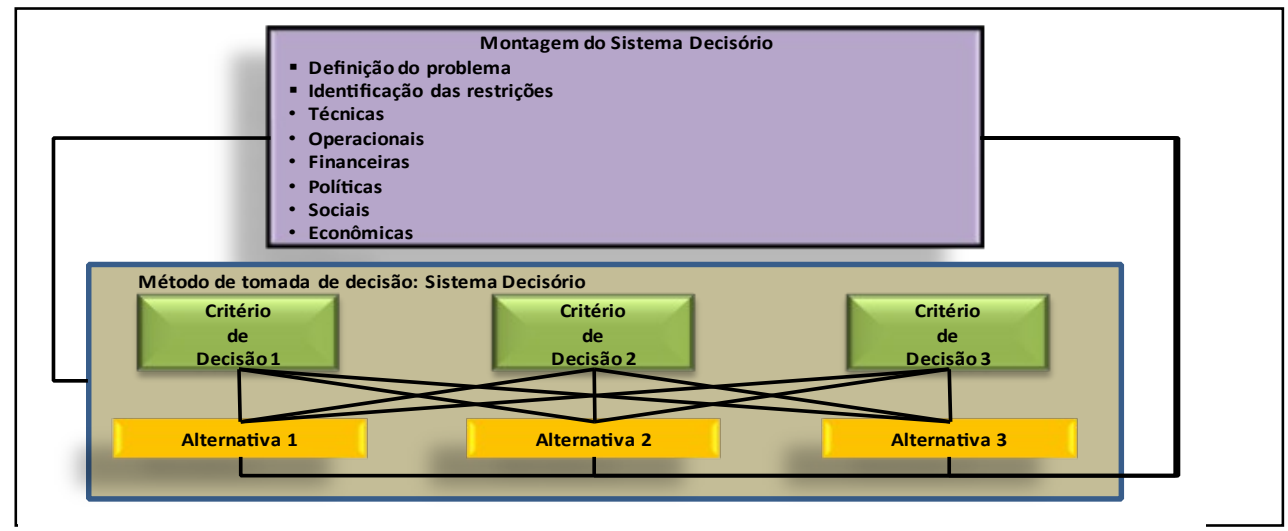

Figura 02- Montagem do sistema decisório nos métodos de tomada de decisão. Fonte: Adaptado pelo autores de (RODRIGUES, 2001).

Conforme ilustrado na Figura 02, a construção esquemática para a resolução de um problema multicritério de tomada de decisão inicia-se com a definição do problema, a identificação das restrições, os critérios e, por fim, as alternativas a serem avaliadas e selecionadas pelo tomador de decisão. Isto ocorre por meio do cruzamento dos critérios com as alternativas e dos critérios com o objetivo final. Para a resolução de problemas que abrangem múltiplos critérios, existem diversos métodos tais como AHP, PROMETHEE, ELECTRE, MAC, TOPSIS, TODIM, (RODRIGUES, 2001). Assim entre as possibilidades existentes de métodos multicritérios de tomada de decisão busca-se à utilização para a escolha de critérios quantitativos e qualitativos de qualidade focando a sua flexibilidade na tomada de decisão, podendo o problema ser definido com intensa participação das pessoas responsáveis pela execução contemplando diferentes interesses.

\subsection{A GESTÃo dOS PROCESSOS PRODUTIVOS E DA MANUTENÇ̃̃o}

A gestão de processos produtivos trata da maneira pela qual as organizações produzem bens e serviços, tudo que se produz chega até você graças aos gerentes de operações que organizam sua produção com auxílio da manutenção. Atualmente, para diferenciar-se da concorrência em termos de qualidade, confiabilidade, serviço, flexibilidade, inovação, ter respostas rápidas às alterações do mercado, a função manutenção tem papel importante nesta tarefa alinhada para tal propósito. A manutenção da planta passou a ser vista de forma diferenciada sendo considerada uma das áreas com papel vital na determinação da produtividade, tendo o objetivo de alcançar variação mínima e manter a planta em boas condições de trabalho ao menor custo possível. No passado era vista como um mal necessário, hoje geradora de lucro (ALSYOUF, 2007). Segundo Alsyouf (2007), os bens não fabricados por um dia perdido devido uma paralisação não planejada nunca será recuperado sem custos adicionais incorridos. De modo geral a manutenção industrial tem 
dois objetivos essenciais; (1) alta disponibilidade de equipamentos de produção e (2) baixos custos de manutenção. Uma vez que estes dois fatores são de natureza contraditória; é essencial otimizar a atividade de manutenção sincronizada com os objetivos dinâmicos da empresa (KARI, 2002). Isso é feito enquadrando ou adotando a estratégia de manutenção correta, que consiste em uma combinação de políticas e estratégias que variam de indústria para indústria (KARDEC; NASCIF, 2013).

O desempenho de uma gestão estratégica de manutenção pode ser analisado através de várias operações de manutenção indicadoras como MTBF, MTTR, produtividade, custo de manutenção, disponibilidade de ativos etc. Os indicadores são calculados com as mudanças analisando os parâmetros acima na estratégia de manutenção implementada (KARI, 2002).

Para Xenos, (2004), a gestão da manutenção esta ligado ao plano de manutenção que é muito importante. A elaboração e o cumprimento deste plano permitirão que a empresa atinja seus objetivos de lucratividade e sobrevivência por meio de equipamentos que não apresentem falhas e que não prejudiquem a qualidade, o custo e a entrega dos produtos, serviços e que não coloque em risco a segurança e a integridade do meio ambiente.

As informações do plano de manutenção devem ser continuamente revisadas com base nos resultados reais das inspeções, reformas, trocas de componentes, realizadas. Além disso, os dados de falhas precisam ser registrados e analisados, por meio de um sistema formal de tratamento de falhas. Os resultados desta análise são outra fonte de informação essencial para a elaboração e revisão periódica do plano de manutenção (XENOS, 2004).

Quando o plano estiver sendo elaborado é possível dimensionar os recursos de mão de obra, materiais, ferramentas, estoque e do tempo de modo a atender exatamente as necessidades de manutenção dos equipamentos. Isso permite otimizar a utilização dos recursos da manutenção sem prejudicar a disponibilidade.

No próximo tópico faz-se uma breve revisão dos conceitos de manutenção, confiabilidade, manutenibilidade e disponibilidade a fim de esclarecer tais conceitos de forma que fique visível o horizonte do estudo proposto.

\subsubsection{O Conceito de Manutenção}

A manutenção é definida como a combinação de ações técnicas e administrativas, incluindo as de supervisão, destinadas a manter ou recolocar um item em um estado no qual possa desempenhar uma função requerida (NBR 5462-1994). Manter significa fazer tudo que for preciso para assegurar que um equipamento continue a desempenhar as funções para as quais foi projetado, num nível de desempenho exigido (XENOS, 2004).

As atividades básicas de manutenção existem para evitar a degradação e prolongar a vida útil dos equipamentos e instalações, causada pelo desgaste natural e pelo uso (FILHO, 2008). Elas estão relacionadas com o tratamento de falhas - detecção, reparo, investigação das causas fundamentais e estabelecimento de contramedidas contra sua reincidência. Num sentido mais amplo, as atividades de manutenção também devem envolver a modificação das condições originais do equipamento através da introdução de melhorias para evitar a sua ocorrência ou reincidência, reduzir o custo e aumentar a produtividade (XENOS, 2004).

O próximo tópico aborda o conceito de confiabilidade dos equipamentos, baseada na manutenção adequada dos ativos.

\subsubsection{Confiabilidade}

Segundo a European Organization for Quality Control (1965) confiabilidade é “a medida da capacidade de um produto operar com sucesso, quando solicitado, por um período de tempo pré - determinado, sob condições ambientais especificas. É medida como uma probabilidade”. A confiabilidade empregada em engenharia de projetos surgiu entre 1939 e 
1945 durante a II Guerra Mundial para o desenvolvimento de sistemas de maior complexidade e que apresentassem uma menor probabilidade de falha durante o período de uso (VILLEMEUR, 1991). Após a guerra, os conceitos de confiabilidade foram aplicados em outros ramos da engenharia que necessitavam uma predição da vida operacional, dos intervalos de manutenção, etc.

No caso de um estudo de confiabilidade em relação a um determinado grupo de falhas, testes de confiabilidade permitem: a melhoria da qualidade; o estudo sobre aspectos relacionados à fixação do período de garantia; ou a reposição de componentes defeituosos de um produto já em produção. Estes testes permitem a obtenção de uma quantidade de elementos suficientes para atender as exigências de critérios estatísticos quanto em compor uma amostra para ensaio de falhas (BASSETTO, NETO e SOUZA, 2006).

Para Marcorin e Abackerli (2001), o domínio da confiabilidade nos produtos confere à empresa uma vantagem competitiva em relação aos seus concorrentes, na pratica, na melhor alocação de custos de garantia e de suporte, inventário de peças de reposição adequado, e melhor custo estendido de seus produtos ao longo do ciclo de vida de cada um.

A probabilidade de ocorrência de falha, representada por $\mathrm{F}(\mathrm{t})$, avalia a possibilidade de um produto apresentar falha no período de tempo ( $\left.0, \mathrm{t}^{*}\right)$ e é representada pela equação:

$$
F\left(t^{*}\right)=\int_{-\infty}^{t^{*}} F(t) \cdot d t
$$

Como um sistema ou está operando adequadamente ou está no estado de falha, a confiabilidade $\mathrm{R}(\mathrm{t})$ pode ser expressa pela relação:

$$
\mathrm{R}(\mathrm{t})=1-\mathrm{F}(\mathrm{t})
$$

Segundo Castro (2009), a taxa de falha $\lambda$ é uma importante função obtida a partir da confiabilidade e é definida como sendo a probabilidade de um item falhar em um próximo intervalo de tempo de operação, dado que o mesmo está operando no início do intervalo, e permite a determinação do número de falhas ocorridas por unidade de tempo, ou seja:

$$
\lambda(t)=\frac{F(t)}{R(t)}
$$

Em que: $\mathrm{F}(\mathrm{t})$ : Função densidade de probabilidade de falha e R(t): Confiabilidade.

Se o comportamento de falha for descrito pela distribuição de Weibull, três parâmetros devem ser considerados: a vida típica $\alpha$, o fator de forma $\beta$ e o fator de escala de tempo Y (MACCHI et al., 2012a).

Quanto à confiabilidade do sistema, ele depende do número de intervenções que são possíveis no horizonte de planejamento. De um modo geral, a confiabilidade do sistema pode ser mantida em alto nível com elevado número de intervenções da manutenção sendo que o fator humano influencia a confiabilidade do sistema, ele está estritamente relacionado ao conceito de manutenção imperfeita, que pode ser aplicado tanto a nível preventivo como corretivo nas políticas de manutenção.

Para Hong (2011), o problema da confiabilidade é um dos mais importantes em projeto de sistemas complexos, é tão importante quanto às características que descrevem o funcionamento do sistema. Um aumento na confiabilidade do sistema pode ser alcançado por um aumento nas confiabilidades de componentes individuais e também pela construção de estruturas especiais com componentes de confiabilidade dada. Mas um velho problema se mantém a tona e importante: determinar por quanto tempo espera-se que um produto funcione dentro de suas especificações, ou o quão confiável é este produto, assim têm-se muitos estudos a serem explorados nesta linha de pesquisa. Em seguida falaremos sobre a disponibilidade que é a capacidade do equipamento atuar perfeitamente em suas funções. 


\subsubsection{Disponibilidade}

Para (MONCHY, 1989), é quando um equipamento disponível está apto para ser utilizado. A partir desta evidencia, a disponibilidade de um ativo é dependente do numero de falhas que ocorrem (confiablidade), da rapidez com que elas são reparadas (manutenibilidade), dos métodos e processos utilizados no exercício das políticas de manutenção, das decisões estratégicas da manutenção e das atividades de apoio efetivas (logística).

Os tempos que influenciam a disponibilidade podem ser classificados como: Tempo total - Tempo em que o ativo poderia ficar disponível para a operação e reflete a capacidade instalada para realizar produção; Tempo Disponível (T) - Parcela da capacidade instalada que pode ser aproveitada para produzir; Tempo não Disponível - Parcelas da capacidade instalada não disponível para produzir, como indisponibilidade por falta de energia, horas locadas para terceiros, os tempos destinados e tempo de espera às intervenções de manutenção preventiva ou corretiva (t). Tempo de Funcionamento - Parcela da capacidade disponível, efetiva utilizada em gerar produção; Tempo de não Funcionamento Representam às paralisações decorrentes de setup, ausência de mão de obra, falta de materiais, programação da produção e uso de velocidade de trabalho menor que a ideal. Observando, têm-se ao longo do tempo normal os tempos disponíveis para gerar produção (T) e os em que o ativo está em manutenção (t), portanto indisponível para a produção. Há diversas formas diferentes de medir a disponibilidade, dependendo de quantas razões para a não operação estiverem incluídas. Quando a “disponibilidade” está sendo usada para indicar o tempo de operação, excluindo a conseqüência da falha, é calculada como segue:

Disponibilidade $(D)=\frac{T M E F}{T M E F+T M D R}$, onde:

TMEF = tempo médio entre falhas da produção ou MTBF,

TMDR = tempo médio de reparo, ou MTTR, que é o tempo médio necessário para consertar a produção, do momento da falha até o retorno em operação.

\section{METODOLOGIA}

Na condução das análises do trabalho, existem dois tipos de dados buscados: publicados e os coletados em entrevistas e observadores no local. O trabalho apresenta-se classificado de acordo com a proposta definida por Gil (2002) como estudo de caso. Além da argumentação lógica, o desenvolvimento do trabalho envolveu uma revisão da literatura citado nos objetivos específicos. E caracteriza-se uma pesquisa qualitativa definida no tema.

Considerando o objetivo dessa pesquisa, a mesma pode ser caracterizada como exploratória descritiva e explicativa. Exploratória por ter como principal finalidade desenvolver, esclarecer, modificar conceitos para a formulação de abordagens condizentes com o desenvolvimento de estudos posteriores. Por esta razão a pesquisa exploratória constitui a primeira etapa desse estudo para familiarizar o pesquisador com o assunto que se procura investigar. É descritiva, no momento que o pesquisador busca descrever a realidade como ela é. Ainda, tem caráter explicativo porque tem a preocupação de identificar os fatores que determinam e que contribuem para a ocorrência de fenômenos (GIL, 2002).

Neste trabalho o estudo de caso da forma como foi apresentado constituiu em um único estudo de caso em uma empresa de terraplanagem no setor de construção civil. A custa da complexidade constituída só será descrita a proposta metodológica no gerenciamento do ativo em questão e na falha estudada. A coleta e análise de dados não se constituem em etapas isoladas estanques, e sim de acordo com Gil (2002), os mesmos estão sempre estreitamente relacionados e são conduzidos numa interação constante.

Para desenvolver o tema proposto, como principais elementos de sondagem, coleta 
e análise de dados foram utilizadas as perspectivas documentais, bibliográficas, relatórios e observações além de visita técnica acompanhada de um responsável da empresa estudada (com possíveis anotações no decorrer do período de produção), como fundamental e importante ingrediente na análise. Assim, o conteúdo do texto é pertinente e útil na medida em que contribui para a solução dos problemas propostos.

\section{RESULTADOS E DISCUSSÃO}

Para exemplificar a proposta buscou-se a definição do equipamento, sendo viável na ocasião o trator de esteiras que será descrito no próximo tópico.

\subsection{DESCRIÇão DA MáQuina EM ESTUdo}

Os tratores de esteiras são equipamentos pesados móveis utilizados em grandes movimentações de terra, de resíduos e na mineração. Possuem potências que podem variar de 80cva até $800 \mathrm{cv}$ e consistem basicamente em um trem de força sobre esteiras com lâmina que empurra o material. O modelo estudado foi de médio porte trator B230 com 243HP, lâmina de $3,73 \mathrm{~m} \times 1,4 \mathrm{~m}$, peso operacional $24.160 \mathrm{Kg}$, seu sistema de transmissão possui 3 velocidades à frente e 3 à ré, e utiliza embreagens de grande diâmetro e alta capacidade, arrefecidas a óleo. A figura 03 ilustra a visão para o objeto de estudo.

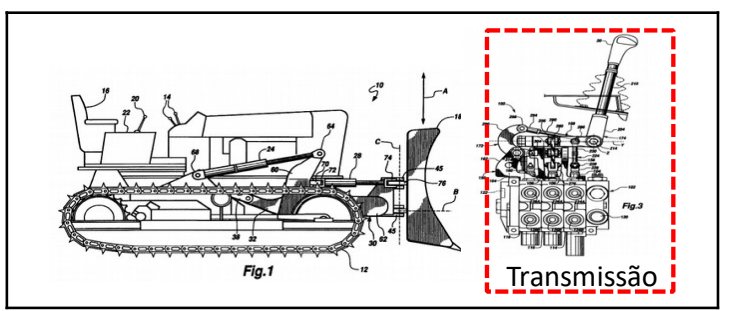

Figura 03 - Sistema de transmissão de tratores esteiras. Fonte: Patente da Compania Komatsu Dresser, 2019.

O trator possui vários conjuntos interligados com possíveis avarias ou falhas, entre as mais criticas está a transmissão, pois demandam maior tempo de reparo, custos e cuidado.

\subsection{DADOS DE FALHAS DO EQUIPAMENTO ESTUDADO}

O problema que motiva este trabalho é a alta incidência de falhas no sistema de transmissão destes tratores de esteiras utilizados em grade obra de terraplanagem. Os dados coletados entre maio de 2017 à outubro de 2018 mostraram que os tratores em análise ficaram parados por falha mecânica $21 \%$ do período útil para produção (aproximadamente 9600 horas - dados de especificação do fabricante). Deste total, 43\% das horas paradas foram devido às falhas na transmissão. Estes dados são referentes às informações de oito tratores de esteiras B230 operando em condições iguais. Levantou-se os dados de horas de trabalho até o momento da falha, data de início da manutenção, descrição da ocorrência, em qual conjunto, liberação do equipamento e o tempo parado.

O total de dias parados por falhas na transmissão dos tratores foram 259 dias como mostra a figura 04, este alto número de falhas no componente chamou a atenção e prosseguindo com as etapas seguintes para o desenvolvimento do modelo. Em todos os equipamentos foram 321 dias parados por diversas falhas.

Todas as falhas no sistema de transmissão foram separadas por tipos de ocorrências e tiveram uma classificação ABC para selecionar as mais críticas, um dos destaques foi o vazamento de óleo na transmissão resultando em 29 dias de equipamento parado, índice muito alto.

Esta falha provoca outras falhas resultando em desgaste, quebra ou trinca de dentes das engrenagens internas, aquecimento excessivo das partes internas da transmissão, entre outros fatores, levando ao colapso posterior do conjunto. 


\begin{tabular}{|c|c|c|c|c|c|c|c|c|c|c|}
\hline & & \multicolumn{2}{|c|}{$\begin{array}{l}\text { EM DIASTRATOR 130TRATOR 237TRATOR } \\
\text { 351TRATOR46TRATOR 538TRATOR } \\
\text { 617TRATOR 7115TRATOR 827Soma Total321 }\end{array}$} & \multicolumn{7}{|c|}{$\begin{array}{c}\text { Dias } \\
\text { ParadosCabine351,22\%Elétrico00,00\% Transmissão } 2 \\
\text { 598,99\% Motor762,64\%Freio642,22\%Material } \\
\text { Desgaste30,10\%Material Rodante60,21\%Total de } \\
\text { dias possíveis288015,38\% d. parado }\end{array}$} \\
\hline $\mathbf{i}$ & $\begin{array}{c}\text { Horímetro } \\
\text { (t) }\end{array}$ & Data entrada & Data Saída & Ocorrência & Conjunto & $F^{\prime}(t)$ & \begin{tabular}{|c|} 
Tempo \\
Parado \\
(dias)
\end{tabular} & $\%$ & ABC & \\
\hline 21 & 1034 & $07 / 08 / 2018$ & $10 / 10 / 2018$ & $\begin{array}{l}\text { REVIISÃO GERAL EM GARANTIA - RETIRADO OS DOIS PACOTES DE GIRO } \\
\text { EESTRUTURA DA CABINE PARA MODIFICACÃO }\end{array}$ & Transmissão & $45,65 \%$ & 64 & 19,94 & A & \\
\hline 34 & 1298 & 01/11/2018 & $30 / 11 / 2018$ & TRANSMISSÃO DANIFICADA & Transmissão & $73,91 \%$ & 29 & 9,03 & A & \\
\hline 39 & 1338 & 01/11/2018 & $30 / 11 / 2018$ & VAZAMENTO DE OLEO NA TRANSMISSÃ̃o & Transmissão & $84,78 \%$ & 29 & 9,03 & A & \\
\hline 42 & 1425 & 01/11/2018 & $30 / 11 / 2018$ & MAQUINA NÃO ENGATA RÉ & Transmissão & $91,30 \%$ & 29 & 9,03 & A & \\
\hline 15 & 908 & $10 / 11 / 2017$ & $29 / 11 / 2017$ & PROBLEMA NO ENGATE DA MARCHA RÉ & Transmissão & $32,61 \%$ & 19 & 5,92 & A & $20 \%$ \\
\hline 31 & 1169 & $18 / 11 / 2017$ & $07 / 12 / 2017$ & TROCA TRANSMISSÃO & Transmissão & $67,39 \%$ & 19 & 5,92 & A & \\
\hline 4 & 330 & $23 / 08 / 2017$ & $10 / 09 / 2017$ & TROCA TRANSMISSÃo & Transmissão & $8,70 \%$ & 18 & 5,61 & A & \\
\hline 18 & 1001 & $16 / 07 / 2018$ & 03/08/2018 & $\begin{array}{l}\text { TROCA DO ÓLEO DA TRANSMISSÃO DENTRO DA REVISÃo DE } \\
\text { GARANTIA }\end{array}$ & Transmissão & $39,13 \%$ & 18 & 5,61 & A & \\
\hline 6 & 527 & $13 / 09 / 2017$ & $30 / 09 / 2017$ & TROCA TRANSMISSÃo & Transmissão & $13,04 \%$ & 17 & 5,30 & A & \\
\hline
\end{tabular}

Figura 04 - Dados de falhas dos tratores B 230. Fonte: Elaborado pelo autor, 2019.

Com os dados coletados elaborou-se os gráficos de probabilidade de falhas F’(t) e confiabilidade $\mathrm{R}(\mathrm{t})$ do equipamento através da formula de Weibull, ilustrados na figura 5 . A curva de probabilidade de falha cresce com o tempo de uso do equipamento em $77,83 \%$ às chances de falha quando o horimetro (t) marcava 1699 horas de uso. Estes dados de crescimento de $\mathrm{F}(\mathrm{t})$ estão fora das especificações garantidas pelo fabricante.

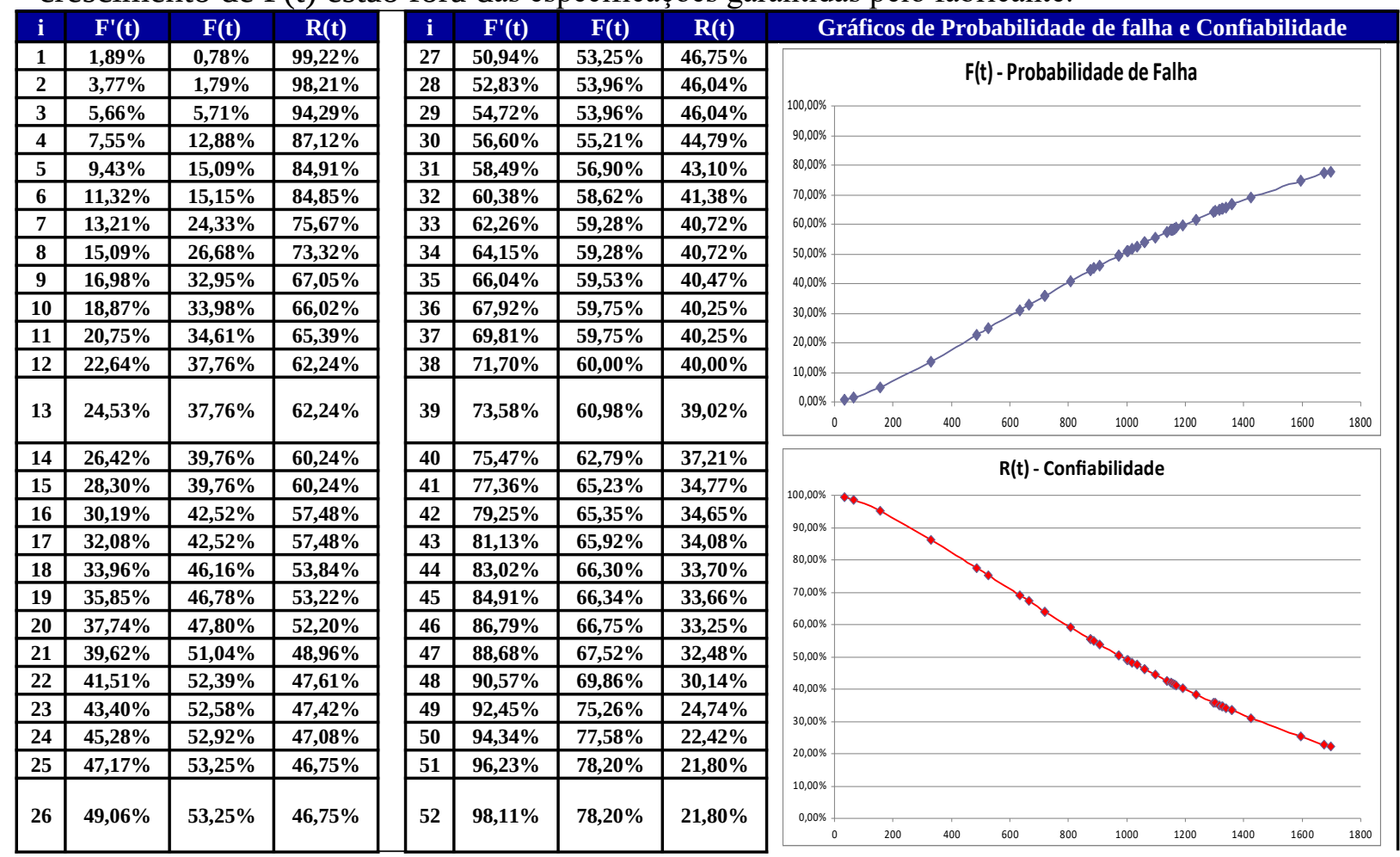

Figura 05 - Tabela de F’(t), R(t), gráfico de Falha e Confiabilidade. Elaborado pelos autores 2019.

Percebe-se que a curva de confiabilidade decresce com o uso continuo do equipamento, indicando 22,17\% no mesmo tempo de 1699 horas, é considerada baixa.

Em seguida realizou-se o levantamento das causas raízes através do diagrama para o vazamento de óleo no conjunto transmissão conforme o modelo proposto. 


\subsection{A Estruturação do Modelo}

As etapas do modelo proposto estão resumidos na figura 6(a), com a aplicação do diagrama de Ishikawa foi levantado às causas raízes da falha critica ilustrado na figura 6(b), em seguida buscou-se conhecimento técnico com a equipe de manutenção, através de pesquisa de projetos, especificações do equipamento, revisão bibliográfica e técnicas para as ações de tratamento sendo encontrado 70 ações para compor os 6M's do diagrama e determinar o conjunto de critérios adequados. Foram 13 critérios estabelecidos pra cada solução das causas raizes: detectabilidade, tempo de realização, custo total, qualidade da atividade, retrabalho da atividade, impacto na disponibilidade, impacto na confiabilidade, performance da máquina, efeito parcial na eficiência de cada ação, efeito global na eficácia de cada ação, resiclagem da ação com o tempo, e transversalidade entre elas. Configura-se assim como problema multicriterio. A terceira etapa foi criar o primeiro cenário do modelo no PROMETHEE, utilizando as ações das causas raízes (em siglas) na figura 6(c) para serem avaliadas pelos critérios. A figura 6(d) mostra a estrutura preliminar do cenário 01.

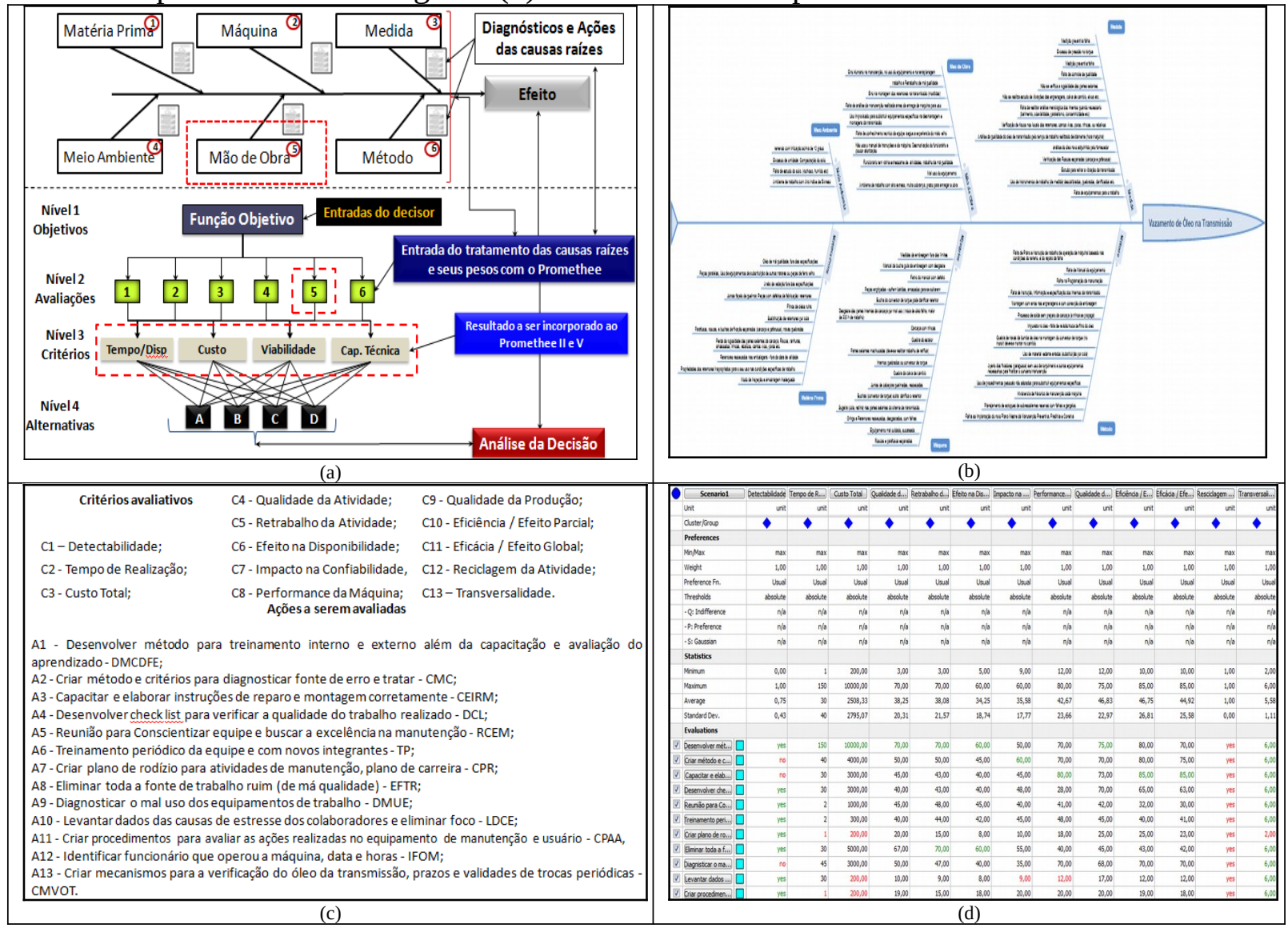

Figura 06 -Etapas para desenvolver o tema proposto. Fonte: Elaborado pelos autores, 2019.

No decorrer dos resultados apresentados pelo Promethee o autor irá analisar os dados das 13 ações obtidas referente ao M (mão de obra) a fim de exemplificar o método proposto, pois o mesmo se tornou robusto com grande volume de informações refinadas e seria inviável analisar todos no espaço deste artigo.

\subsubsection{Análise dos resultados do Modelo no PROMETHEE}

A classificação parcial das alternativas é determinada de acordo com o método difuso Promethee I. Basicamente, quanto maior o fluxo de saída ( $\boldsymbol{\Phi}+$ valor) e menor o fluxo de entrada ( $\Phi$ - valor), melhor o material. De acordo com esses valores, o ranking parcial do cenário um, as ações "Desenvolver método para treinamento interno e externo além da capacitação e avaliação do aprendizado - DMCDFE”; “Criar método e critérios para 
diagnosticar fonte de erro e tratar - CMC"; "Capacitar e elaborar instruções de reparo e montagem corretamente - CEIRM"; "Eliminar toda a fonte de trabalho ruim (de má qualidade) - EFTR"; "Diagnosticar o mau uso dos equipamentos de trabalho - DMUE"; "Desenvolver check list para verificar a qualidade do trabalho realizado - DCL" foram as que mais se destacaram e apontadas como fluxos positivos, por outro lado as ações "Criar plano de rodízio para atividades de manutenção, plano de carreira - CPR" e "Criar mecanismos para a verificação do óleo da transmissão, prazos e validades de trocas periódicas - CMVOT” são as piores alternativas e ilustrados pela figura 07(a).

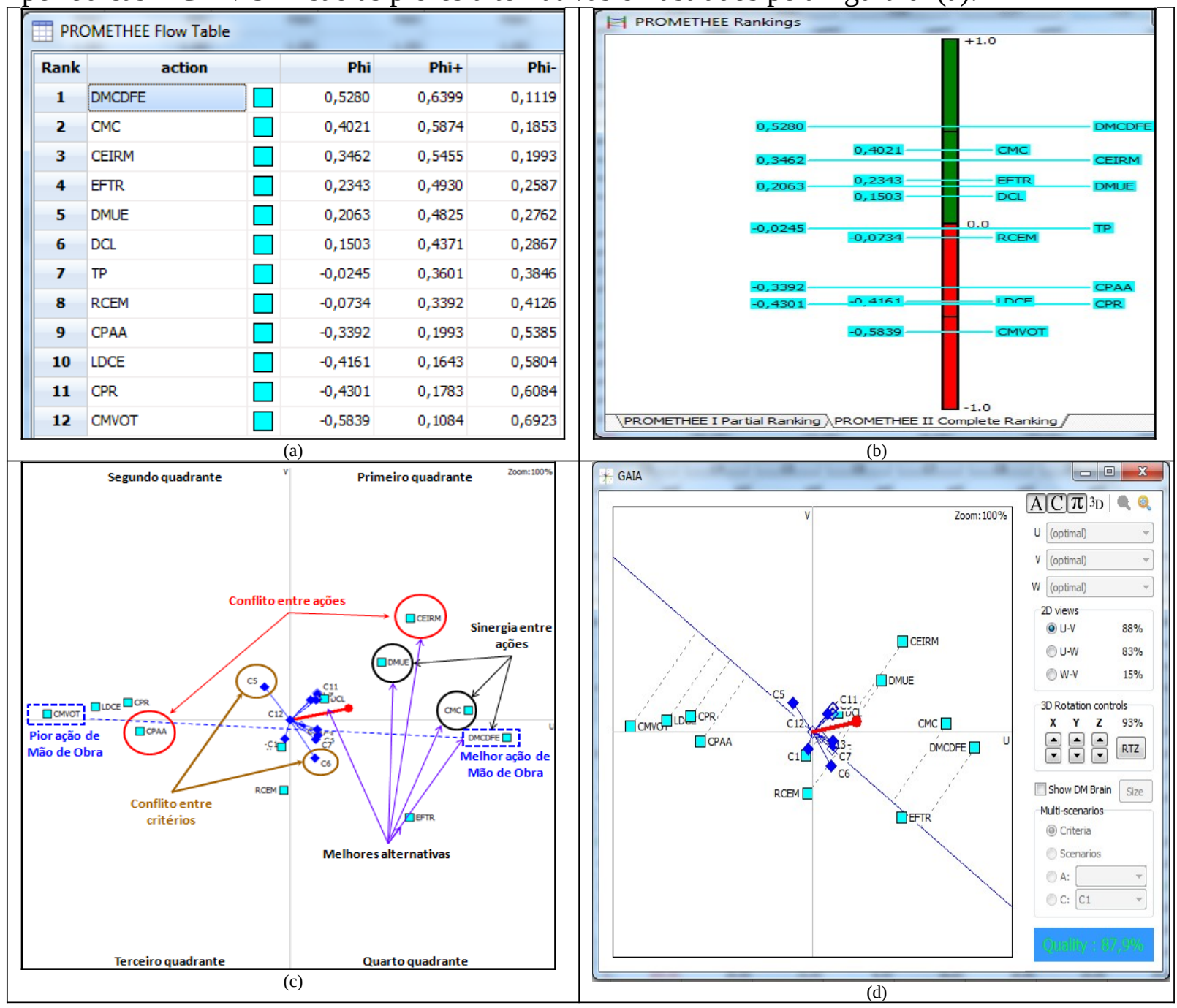

Figura 07 - Análise das ações para (mão de obra) da falha estudada. Fonte: Elaborado pelos autores, 2019.

Já o PROMETHEE II Complete Ranking ilustrado na figura 7(b): realiza a ordenação de forma decrescente completa baseada no fluxo líquido (Phi) calculado pela diferença entre os fluxos de importância positivo e negativo. Com a ajuda do ranqueamento no Promethee II, na figura 7(b) observa-se que três grupos de ações aparecem claramente sendo 1) A Ação DMCDFE tem um escore Phi maior, neste grupo é composto pelas ações CMC, CEIRM, EFTR, DMUE e por fim a ação DCL; 2) TP e RCEM têm pontuações negativas e são todos muito próximas de zero. Elas são compostas por ações cotidianas e realizadas com mais freqüência; 3) As ações CPAA, LDCE, CPR e CMVOT também têm pontuações muito próximas, são mais negativas e distantes de zero e estão na parte inferior.

No Visual PROMETHEE V GAIA, existem três dimensões a serem calculadas: U é o primeiro componente principal, contém a quantidade máxima possível de informação, $\mathrm{V}$ é o segundo componente principal, fornecendo a informação adicional máxima ortogonal a U, 
W é o terceiro componente principal, fornecendo a informação adicional máxima ortogonal a ambos U. Os controles de visualizações 2D permitem alternar entre três visualizações 2D: U$\mathrm{V}$ : Esta é a melhor vista 2D possível. No trabalho apresentado, ele reúne $88 \%$ das informações; consideradas bastantes confiáveis. U-W: Com relação à visão $U-V$, ela é vista de lado (W é ortogonal a ambos os U e V), é de baixa qualidade e reúne apenas 83\% das informações. W-V: Com relação à visualização U-V, ela é vista de cima, é a visualização 2D de menor qualidade disponível no Visual PROMETHEE e reúne aqui apenas 15\% das informações; os dados estão ilustrados na figura 7(c) e 7(d).

A solução indicada no PROMETHEE V diz que as ações ótimas a serem observadas e desenvolvidas pela gerência ou outros decisores envolvidos na gestão da manutenção da organização são: a) Desenvolver método para treinamento interno e externo além da capacitação e avaliação do aprendizado - DMCDFE; b) Criar método e critérios para diagnosticar fonte de erro e tratar - CMC; c) Capacitar e elaborar instruções de reparo e montagem corretamente - CEIRM; d) Desenvolver check list para verificar a qualidade do trabalho realizado - DCL; e) Eliminar toda a fonte de trabalho ruim (de má qualidade) EFTR; f) Diagnosticar o mau uso dos equipamentos de trabalho - DMUE.

\section{CONCLUSÕES}

A metodologia desenvolvida de análise de decisão proposta é considerada inovadora, pois de acordo com o conhecimento dos autores não existe tal encadeamento de ferramentas na literatura. O conjunto diagrama de Ishikawa e o método PROMETHEE incluem muitas outras possibilidades de análise possível sendo um modelo poderoso e excelente para a análise de decisão com a possibilidade de aplicações nas diversas áreas industriais, governamentais, sociais entre outras. Com um bom entendimento de aplicação dos pesos e os valores para as ações pode-se chegar a uma escolha coerente das decisões a serem tomadas em busca de um objetivo comum, seja pelo gestor, departamento, organização ou no encontro do melhor caminho. Percebe-se a oportunidade de estudos com o Ishikawa e o PROMETHEE para uma decisão de manutenção centrada em confiabilidade como fator gerador de novos conhecimentos no planejamento manutenção de forma efetiva, com uma forma de análise aprofundada até a raiz do problema, na qual inclua a verificação dos demais conjuntos do equipamento.

\section{REFERÊNCIAS BIBLIOGRÁFICAS}

ALSYOUF, Imad. “The Role of Maintenance in improving Companies' productivity and Profitability” Int. J. Production Economics, 2007, 105: 70-78

BEN-DAYA, M.; S.O. Duffuaa, (1995). "Maintenance and quality: the missing link", Journal of Quality in Maintenance Engineering, Vol. 1 N0. 1, p.20-26. Doi: https://doi.org/10.1108/13552519510083110.

BRANS, JEAN-PIERRE, MARESCHAL, BERTRAND (1994) “The Promethee-Gaia decision Support System Multicriteria Investigations”. Investigacion Operativa, 4, p.107117.

CASTRO, I.T., (2009). A model of imperfect preventive maintenance with dependent failure modes. European Journal of Operational Research, 196(1), pp.217-224. Available at: http://dx.doi.org/10.1016/j.ejor.2008.02.042.

DOBRUSSKIN, Christoph. (2016). On the identification of contradictions using Cause Effect Chain Analysis. TFC 2015- TRIZ FUTURE 2015. Procedia CIRP 39, p. 221 - 224. 
DUPHILY, Ronald. Root Cause Analysis Best Practices Guide (2014). AEROSPACE REPORT NO. TOR-2014-02202: available on the web at http://www.aerospace.org/wpcontent/uploads/2015/04/TOR-2014-02202-Root-Cause-Investigation-Best-Practices Guide.pdf

GIL, ANTONIO CARLOS. Como Elaborar Projetos de Pesquisa. 4. ed. São Paulo: Atlas, 2002.

GOMES, L.F.A.M.; GOMES, C.F.S. Tomada de decisão gerencial: enfoque multicritério. São Paulo: Atlas, 2012.

HONG, Wiliam. APLICAÇÃO DO MÉTODO DE ANÁLISE DE RISCO AO ESTUDO DO DERSCARRILHAMENTO. 2011. 194 p. Tese (Mestrado em Engenharia Mecânica de Projeto de Fabricação) - Escola Politécnica da Universidade de São Paulo. São Paulo, 2011.

ISHIKAWA, K. Controle de qualidade total: à maneira japonesa. Rio de Janeiro: Campus, 1993.

KARDEC, A; NASCIF, J. Manutenção: Função Estratégica. 4. ed. Rio de Janeiro: Qualitymark, 2013

KARI, Komonen. "A cost model of industrial maintenance for profitability analysis and benchmarking”. Int. J. Production Economics, 2002, 79: 15-31

LORENZO, Donald K.; JACKSON, Laura O. et al. Root Cause Analysis Handbook (2008). 3rd edition, ABS Consulting.

MACCHI, M., GARETTI M., CENTRONE D., FUMAGALLI L., PAVIRANI G.P., 2012a. Maintenance management of railway infrastructures based on reliability analysis. RELIABILITY ENGINEERING \& SYSTEM SAFETY, 104, pp. 71- 83.

MARCORIN, A. \& ABACKERLI, A. (2001) - Estudo Exploratório Sobre Áreas Potenciais de Aplicação de Técnicas de Confiabilidade. Anais do XXI ENEGEP. Salvador.

MONCHY, François. A Função Manutenção - Formação para a Gerência da Manutenção Industrial. São Paulo: Durban, 1989.

RODRIGUES, F. H., MARTINS, W. C., \& MONTEIRO, A. B. F. C. (2001). O Processo de Decisão Baseado em um Método de Análise Hierárquica na Tomada de Decisão Sobre Investimentos. In J. V. Caixeta Fo. \& R. S. Martins (Eds.), Gestão logística do transporte de cargas. São Paulo: Atlas.

ROY, B. (1985). Méthodologie Multicritère d’Aide à la Décision. Economica, Paris.

SAATY, T. L., \& VARGAS, L. G. (2012). Models, methods, concepts \& applications of the analytic hirarchy process (2 ed., International Series in Operations Research \& Management Science, 175). New York: Springer. 345 p.

SLACK, NIGEL; CHAMBERS, Stuart; JOHNSTON, Robert. Administração da produção. 3. ed. São Paulo: Atlas, 2009.

VILLEMEUR, Alian. Reability, Availability, Maintainability and Safety Assessment. V.1, $1^{\text {st }}$ ed. New York: John Willey \& Sons, 1991, p. 363p.

VINCKE, P. (1992). Multicriteria Decision-Aid. Bruxelles.

XENOS, HARILAUS G. Gerenciando a Manutenção Produtiva: O caminho para Eliminar Falhas nos Equipamentos e Aumentar a Produtividade. Nova Lima: Editora Falconi, 2004. 